\title{
Doing Consciousness Studies at Goddard College
}

\author{
FRANCIS X. CHARET \\ Goddard College \\ Francis.Charet@Goddard.edu
}

\author{
HILLARY S. WEBB \\ Ph.D. Candidate, Saybrook Graduate \\ School and Research Center \\ hillary@hillaryswebb.com
}

\section{ABSTRACT}

In the first part of this article we briefly describe the design and development of a Consciousness Studies concentration at Goddard College, a student centered, progressive educational institution in the northeastern United States. We emphasize the tensions we experienced between different orientations in Consciousness Studies and especially the one related to the scientific and transpersonal ends of the spectrum of consciousness. In the second part, we relate the scientific-transpersonal issue that we experienced at Goddard to the broader theory and practice of Consciousness Studies. We also link the latter to the so-called explanatory gap between third and first person forms of consciousness and the call for first person methods of exploring consciousness. Our conclusion is that the scientific approach has the edge and is at risk of assimilating other approaches to the detriment of Consciousness Studies. In our view, the future development of Consciousness Studies, both in theory and practice, requires several different approaches from the scientific to the transpersonal and privileging one is to narrow the field of possibilities and to risk falling prey to one-sidedness. KEYWORDS: anthropology of consciousness, consciousness studies, education, science, transpersonal theory.

\section{INTRODUCTION}

While once buried within the more traditional disciplines such as philosophy, psychology, religious studies, neurobiology, and the like, in recent years, consciousness studies has begun to emerge as an independent field within several

Anthropology of Consciousness, Vol. 18, Issue 1, pp. 51-64. ISSN 1053-4202, (C) 2007 by the American Anthropological Association. All rights reserved. Please direct all requests for permissions to photocopy or reproduce article content through the University of California Press's Rights and Permissions website, http://www.ucpressjournals.com/reprintinfo/asp. DOI: 10.1525/aoc.2007.18.1.51 
educational institutions across the United States and abroad. One such institution currently in the midst of exploring this somewhat new territory is Goddard College, located in Plainfield, Vermont. In the following article we will bring together two things: (1) the progressive education model of student-centered, integrated learning that informs the pedagogical practices of Goddard College and the design and development of a graduate concentration in Consciousness Studies and (2) some additional reflections on where we think more needs to be done to effectively sustain consciousness studies as a legitimate and important area of study and/or discipline. We have also tried to weave into these our two perspectives, one of a recent graduate (HSW) and the other of the concentration's coordinator (FXC). We feel quite strongly that the lines of development of consciousness studies at Goddard have proven to be quite valuable for both student and faculty and, as a model of progressive pedagogy and a window on where we need to go, it might be of interest to others in the business of "doing consciousness studies."

We would like to open by offering for our readers' consideration a few statements as a way of clarifying a number of our assumptions. The first is that we have come to believe that, broadly understood, consciousness studies is not something new. That is to say, it is not something that has been invented by zoth-21st century Western culture, but rather is a field of study that humankind has engaged in since we first began to reflect upon our own subjective processes and their relationship to the greater universe. The second is that the two of us, as well as many others who have participated in the creation of a graduate concentration in Consciousness Studies, have been subject to a variety of experiences that have influenced our assumptions and conclusions about what should go into the making of consciousness studies as an independent area of studies and/or discipline. For example, both of us have had the privilege of having been in contact with nonWestern cultures, which has led to experiences that have dramatically altered our conceptions of what the world is and our place within it. One of us (HSW), has for several years been involved in the study of shamanic traditions, and the other (FXC), has immersed himself in a number of religious cultures in both scholarly and personal ways. Both of our experiences, as well as the experiences of other students and faculty, have been crucial to the development of the graduate concentration in Consciousness Studies at Goddard College.

The inclusive history and prehistory of consciousness studies to which we have alluded has yet to be reconstructed and assimilated into the general overview of this emerging discipline and will, hopefully, be undertaken some day. Suffice to say it will have to involve something more than the usual tracing back of a scientific perspective to contemporary and classical Western sources and, instead and more legitimately, involve the myriad expressions of what can be termed a preoccupation with consciousness, in its various linguistic and cultural forms, right across the timeline and geographical landscape of the planet. In spite of the efforts of some, we are still distant from accomplishing this task. The final point 
that we would like to make is the recognition that, given the nature of consciousness studies as a field that requires the inclusion of a number of ways of knowing, it will no doubt continually change in accordance with the institutions at which it is taught, the particular faculty teaching it, and the students who are engaging in this area of study. In what follows we offer nothing more than a modest and provisional attempt to make due with what we have available to us so far but that we feel is reflective of this broader perspective on "doing consciousness studies."

PROGRESSIVE PEDAGOGY AND CONSCIOUSNESS STUDIES

Goddard College is a leading progressive educational institution in the northeastern United States. It has a long and checkered history of student-centered, experiential learning, radicalism, and social activism that has often brought it to the attention of other institutions and the public at large. In recent years, the college has undergone a transformation in order to adapt to the current educational market of focused adult learning without sacrificing its fundamental commitment to student-centered, progressive education that emphasizes interdisciplinary studies and "thinking outside of the box." While for many years, Goddard was primarily a residential college, the on-campus program was phased out in 2002 and a series of undergraduate and graduate low-residency programs now constitute the curriculum with a current student population of approximately 600 drawn from across the United States and elsewhere and with the expectation that this will level off at 1,000 in the next few years.

For those unfamiliar with the low-residency educational model, this means that students attend week-long, on-campus residential programs at the beginning of every semester, during which time they meet with faculty advisers to discuss the subject matter and material that they wish to address during the upcoming semesters. Students typically come to the program with an idea or topic that they wish to explore, and it is the adviser's role to help the student round out their original ideas. During residencies, students have the opportunity to attend presentations on a variety of subjects given by the faculty, fellow students, and guest lecturers. After the residencies, students return to their respective communities and begin the work that they have outlined with their adviser. In the master's program, of which the Consciousness Studies concentration is a part, students submit five packets of written work to their adviser over the course of the semester. The adviser reads each submission and then responds with questions, comments, and ideas for further direction and dialogue. In the M.A. program, students typically spend four to five semesters immersed in the subject(s) of their choice, and their studies culminate in a thesis or commensurate final product as approved by the faculty.

During the period of transformation in which Goddard went from a largely residential to a non-residential college, there emerged a number of areas that were identified as best expressing students' interests. We on the faculty and in the administration listened to the concerns of students who felt that there was no 
place for them to go to study topics that emphasize the more transpersonal and experiential dimension of consciousness studies; topics that were close to their hearts, but that did not fit into the materialist view of consciousness found at most traditional universities. For example, one of us (HSW) had attended "Theological Education Day" at a nearby Ivy League college, and was shocked to discover that no experiential work was required or encouraged of students studying the teachings of the world's religions. Given this apparent vacancy in many college curriculums, and given its momentum as an emerging field, the consciousness studies concentration was born at Goddard. It became a concentration within the Individualized M.A. program in 2004. ${ }^{1}$

Initially, what we attempted to identify as students' interests that we could relate to the emerging area of consciousness studies was informed by a sense of it including, if not highlighting, the first person, experiential approach, with an emphasis on the transpersonal dimension of consciousness. This broad designation especially included spiritual and psychotherapeutic traditions and practices that range from shamanism to Buddhist meditation to Jungian and transpersonal psychology, with just about everything that can be imagined in between. As the result of surveying the institutions and programs that have developed consciousness studies as an area, it became clear that there were roughly two kinds: (1) the more conventional programs that emphasize the objective scientific approach to the study of consciousness and (2) more alternative programs that emphasize the subjective, experiential, and transpersonal perspectives. To the students and faculty at Goddard, we came to see that both had attractions that we felt we would need to include in our concentration. The dilemma we faced then was the degree to which either side of the spectrum seemingly opposed or, at the very least, excluded or ignored the other. In other words, it seemed you could have one or the other but not both, and we wanted both!

Our motivation for this had less to do with the fact that we thought these two perspectives could be somehow easily reconciled than that we were attracted by a conception of integration that is at the heart of the progressive education model and required we get it all in there if we possibly could. This meant that both students and faculty would have to grapple with the tensions and contradictions within each and in relation to each other. This idea was immensely exciting to us. In fact, we felt very strongly that because the relationship between the objective-scientific and subjective-experiential-transpersonal was obviously a contentious factor in the broad field of consciousness studies, we would need to be as inclusive as possible, even if our students tended in one direction more than another. Over the course of many discussions between faculty and students, we realized that an integrated model of consciousness studies would need to move beyond the polarized conception of consciousness studies as a spectrum of theories, ideas, and opinions ranging from the scientific to the transpersonal, from reductionism downward to reductionism upward. Further exploration of the range of work being done in consciousness studies added other important 
dimensions to the way we began to conceptualize it as an area of study and practice, and these included the social sciences, the arts and the humanities. All these fields have contributed substantially to consciousness studies and offered us additional perspectives, softening the polarization that the scientific-transpersonal tension surfaced. We eventually included all these in a model of the four "core learning areas" that has since stood for what the Consciousness Studies concentration looks like at Goddard. They are outlined in our literature and on the Goddard web site as:

(1) The Scientific and Philosophical Study of Consciousness: The scientific and philosophical approach to the study of consciousness can be highly technical and intellectually demanding, drawing on disciplines from the neurosciences to the philosophy of mind. As part of the field of Consciousness Studies, it is important to have an overview of the ongoing work being done in these areas.

(2) The Social Scientific Study of Consciousness: Various social scientific disciplines have provided material and additional perspectives on the study and exploration of consciousness. Chiefly, these have been psychology, sociology, and anthropology and have played a crucial role in highlighting the psychological, social, and cultural contexts in studying and understanding consciousness.

(3) The Transpersonal Study of Consciousness: The term transpersonal covers a range of meaning that is evident in the prefix "trans" and refers not only to "beyond," but also "across," "through," "pervading," and "directed towards change or transformation." The transpersonal approach involves many fields such as religious studies, Jungian, and transpersonal psychology, as well as deep ecology.

(4) The Arts and Humanities: Both the arts and humanities provide content and a perspective that has shaped the way humans give expression to various forms of consciousness. In particular, the visual arts and literature provide additional sources for the study and exploration of consciousness from the symbolic and imaginary to the aesthetic.

[www.goddard.edu]

In addition to encouraging broad coverage in all these areas, a program requirement at Goddard emphasizes the need to address the degree criteria that we have described in a short hand way as: "knowing" (academic and intellectual knowledge of the given subject[s]), "being" (how the subject relates to the student's life, outer and inner) and "doing" (how the student can bring their learning into the larger community for positive transformation). In order to support students in fulfilling these requirements each student must (1) write an "identity essay" that conveys in academic and personal terms their relationship to the field 
of consciousness studies and (2) undertake and document an "engaged practice" in the community that reflects their newly acquired knowledge. Examples of the latter that students have accomplished are the setting up of a retreat for gay and lesbian youth to consider their spiritual concerns, drawing on models of exploration and integration from transpersonal psychology, a workshop guiding participants in a yoga practice, and so on. Finally, the student's work culminates in an integrated final product or thesis that expresses the accomplishments of the student and embodies a synthesis of the aforementioned "knowing," "being," and "doing." The substance of this final product is then presented to the Goddard community during the graduating residency. By doing so, not only do the other students and faculty at the college have an opportunity to benefit from the student's work, but it is often by articulating their work in this way that the student begins to recognize the learning that has taken place over the course of the semesters and helps ready them to step confidently into the conversation taking place in the greater context of the field and bring their work into the world.

Residencies at Goddard provide a forum in which Consciousness Studies students of different persuasions can come together to discuss their work and exchange ideas. This has turned out to be an integral part of the students' development, for it is here that many of them first become exposed to the variety of disciplines and perspectives within the field. Furthermore, there is a sense of community that occurs throughout this week, both with students and faculty. One of the major complaints that students have is that in their "real lives" they have few opportunities to discuss these issues with friends and family members. Oftentimes, they are faced with having to explain and even defend this somewhat atypical field that they have decided to enter into. Residencies provide the opportunity to develop support structures and idea sharing, with the other students, the faculty (who, as academic generalists have a wide knowledge base), as well as guest speakers - professionals in the field who give lectures and have discussions with the students.

Consciousness Studies students come to Goddard as adult learners with diverse backgrounds, differing levels of experience, and a variety of personal and professional inclinations. When faced with the "knowing," "being," and "doing" criteria, students usually feel more comfortable with one or two of these three lenses through which they are asked to view their work. For example, a student may feel comfortable and confident dealing with the more intellectual and theoretical aspects of intellectual knowing. For this kind of student, the research and design of a body of work comes easily, but what may be more elusive is how to bring these abstract concepts concretely into the world by finding a means of "doing" them. Or, prior to coming to Goddard, students may have developed a certain set of beliefs and practices that they have integrated into their life, but these beliefs have become so much a part of the students' "being" that they may not be able to articulate them on an intellectual level. Or, even more challenging, 
such students may have become so invested in a certain set of ideas as a way of life that critical self-reflection and examination of their ideas does not come without a sense of their own core self becoming painfully deconstructed. It is here that the faculty is an integral part of the students' development, and advisers are tasked with challenging the students to step out of their comfort zones and into this broader territory that is the field of consciousness studies. A student with very solid and firmly developed spiritual leanings may be asked to write a critical review of publications that considers the more materialist theories of consciousness. Or, conversely, a student with an interest in neuroscience may be encouraged to engage in some sort of introspective or spiritual reflection or practice. The two types representing paradigms of reductionism upward and reductionism downward are both asked to consider their opposites and, finally, to either find some common ground between the two or, at the very least, acknowledge each other's perspectives as legitimate ways of knowing and be able to co-exist with some kind of respect, even if it is begrudging respect! Needless to say, this can be a very challenging process, both for the student and for the adviser, and yet our experience has shown that the students who take the opportunity to consider the validity of alternate viewpoints and the limitations of their own ideas and experiences invariably walk away feeling more competent and more able to enter into the conversations occurring at many levels of the field of consciousness studies.

The thesis topics of graduate students in Consciousness Studies reflect the diversity of subject matter to be found within the Consciousness Studies concentration. One student, for example, wrote a substantial thesis that could broadly be designated as a work in philosophy of mind that incorporated extensive discussion of elements of esotericism and mysticism. Another did an intensive study and engaged in and documented the practice of yoga in the tradition of Kashmir Shaivism. Another undertook a cross-cultural examination of how various spiritual, philosophical, and psychological traditions seek to overcome paradox and duality using an interdisciplinary approach that included anthropology of consciousness, philosophy of mind, Jungian psychology and religious studies. Others have worked with dreams and Jungian psychology, Buddhist meditation and approaches to peace and social activism, death and dying, and so on.

At graduation, students are bound to ask themselves: what am I going to do with this degree in Consciousness Studies? While some have employment prospects, other graduates often find they are in the position of needing to create their own jobs and develop their own opportunities, such as designing and presenting workshops within their communities. Those who hope to teach at the university level go on to Ph.D. work at similar institutions. In other words, there is a narrow field of employment opportunities at this point for individuals with degrees in Consciousness Studies. Where both Goddard's Consciousness Studies concentration and the field of consciousness studies in general could be improved is helping students explore the possibilities for future work within this 
field. We have found that part of the reason students may not be aware of what is going on in the rest of the consciousness studies field is that they don't know how they themselves fit or could potentially fit into the field beyond the safe "womb" of Goddard. We are aware that advisers and students should perhaps spend more time discussing the possibilities for the student's future, outline options that exist - such as writing for academic journals or popular magazines, various alternative forms of employment, research and grant writing, teaching opportunities and so on. Often, however, students' work in Consciousness Studies at Goddard constitutes a labor of love, rather than a calculated means of advancing their careers. Goddard provides an opportunity for students to take the time to work in an educational environment that is supportive of their interests and experiences and provides resources and feedback to further develop them in a meaningful way.

In summary, at Goddard we have evolved a concentration in Consciousness Studies that has, at its heart, a conception of the field that requires various approaches to create an integrated understanding and experience, and so far this has served us all well. Looking at other institutions and programs, and also across the field at the various publications, web sites and conferences, we are left with a sense that we have, in our own modest way, grappled with some of the salient issues that have come to the fore in consciousness studies. In what follows, we would like to attempt to address what we feel has emerged as important theoretical issues that are implicit in our experience of the doing of consciousness studies.

HURDLES, METHODOLOGICAL ISSUES AND COMPROMISES

What we have presented so far is a broad outline of some of the elements that went into the making of the Consciousness Studies concentration at Goddard. Much more detail could be provided and other aspects could be discussed in an extended way. Nevertheless, it seems to us, drawing on our own experience working in consciousness studies, there is a matter that invites further comment that has surfaced in the previous presentation and is widely present in the theory and practice of consciousness studies. This is the tension between the conventional scientific orientation to consciousness studies and the alternative one that emphasizes the inclusion of the transpersonal. Seemingly related to this is the issue of the so-called "explanatory gap" between third- and first-person consciousness that occupies much recent discussion. This tension and issue not only pervades the literature, it takes institutional forms in university departments and centers, as well as in conferences, symposia, and associations. In our view, as we have stated, to exclude either end of the spectrum of consciousness studies is a shortcoming that will adversely affect the "doing of consciousness studies." The fact is that as the enormous authority of science in our culture has given it the edge, there is a danger that other alternative approaches will be excluded or 
assimilated where the scientific one is emphasized. If this happens, we believe it will be to the detriment of students and consciousness studies in general.

By way of example, an editorial on "The Future of Consciousness Studies" only a few years back in the Journal of Consciousness Studies bears this out in the crude, but not unrealistic, division between areas that were considered "kosher" such as philosophy, neurobiology, and cognitive science versus "taboo" areas such as anthropology, anomalies, religion, psychotherapy and transpersonal psychology (Editor 1997:285). And while there are indications that alternative perspectives to the scientific one are now being included in such major forums as the Tucson "Toward a Science of Consciousness" conferences, where we note the recent inclusion of references to anomalous phenomena or reporting on the study of Tibetan meditators, it also is usually accompanied by such caveats as:

Research on meditation needs to bracket the metaphysical claims - since they are not scientifically tractable now.

[Faw 2006:97; also Whitehead 2004]

We note, too, there are concessions on the other side as in a recent and informed book on science and spirituality by the Dalai Lama entitled The Universe in a Single Atom. In it he states:

If scientific analysis were to conclusively demonstrate certain claims of Buddhism to be false, then we must accept the findings of science and abandon those claims.

[2005:3]

Yet, admirable as this tolerance and graciousness is, it nevertheless still confers on science a position of authority and even sovereignty over the entire field of consciousness studies that, we feel, begs for examination. While this would require a far more extensive discussion than can be engaged in here, we would like to raise a few concerns that we hope our readers will find stimulating and helpful. Even a cursory glance at the recent growing literature on consciousness yields the impression that for all the remarkable achievements of science, there is growing consensus that it has to date come short of adequately addressing the so-called "hard problem" of accounting for phenomenal experience and bridging the now famous "explanatory gap" between first-person and third-person consciousness. The scientific approach is determinedly third person and has achieved remarkable feats in its bid for objectivity but has not come up with a satisfactory explanation for what causes first-person experience of consciousness. It would seem to be reasonable, given that subjective experience is essential to consciousness, to suggest that the neglected avenue that needs to be explored is the experience of first-person consciousness. This is by no means an easy task (Varela and Shear 1999).

To undertake to develop a rigorous methodology of first-person empiricism is a notoriously difficult challenge, much because of how firmly entrenched is 
the idea that objectivity and the conventional scientific method are the normative assumption and means of attaining some degree of certitude. Yet, arguably, without first-person input, we are in danger of accomplishing little more than measuring and re-measuring ever more precisely the outside of the fishbowl of consciousness. And while arguments have been made about the limitations of introspection for the attainment of insight, the fact remains that there are traditions of disciplined introspective empirical analysis that have been built up in various cultures over the ages and attained a remarkable degree of understanding about the geography of the inner landscape of consciousness. These mental disciplines and contemplative practices, respectfully learned, could be of some use in exploring and excavating the inner reaches of consciousness. Indeed, the Dalai Lama himself has called for just this:

To some degree I think the experience of, indeed, training in, some of these techniques of mental discipline (or others like them) will have to become an integral part of the training of the cognitive scientist if science is serious about gaining access to the full range of methods necessary for a comprehensive study of consciousness.

[2005:160]

In fact, it seems clear that most cultures have evolved techniques of disciplined introspection, and some have developed these into remarkably sophisticated systems of practice with a capacity to bring about measurable changes in consciousness with positive physical and psychological effects. Many of our students who engage in such practices claim the same, and there is a growing literature that documents this accomplishment and has attracted widespread attention (Murphy et al. 1997). And in the West, techniques have emerged that have contributed to the mapping of the inner realm, such as Jungian and transpersonal psychology, both of which are contemporary methods of disciplined introspection and analysis, largely ignored or dismissed by current neuroscience and those working in the philosophy of mind (Cortright 1997). The rationale in favor of the Dalai Lama's invitation that scientists become trained in mental disciplines or contemplative practices could be extended to all students of consciousness studies, if there is sufficient motivation to include in the study of consciousness an empirically grounded first-person perspective, something that now seems to be receiving growing attention. If this is pursued, in the course of time, a sufficient amount of accumulated experience and data could make a significant contribution to understanding the phenomena of consciousness.

Positive as all of this seems, it is also necessary to add some qualifications and even reservations and these have to do with our concern with the degree to which the scientific model remains implicit in the proposal of supporting first-person 
empirical approaches to the study of consciousness. We must not forget that there are other, equally valid ways of knowing. As Gadamer has reminded us:

The human sciences are joined with modes of experience which lie outside of science: with the experiences of [religion], philosophy, of art, and of history itself. These are all modes of experience in which a truth is communicated that cannot be verified by the methodological means proper to science.

[1988:xii]

Undue privileging of the first-person perspective and especially assimilating this to a scientific model seems to us to risk creating more problems than it solves and not least of these are the constraints that come, once again, from limiting knowledge and truth to the control of the scientific method. In the case of transpersonal or religious events, it has (1) the unfortunate consequences of reducing the transpersonal and spiritual to personal experience, uncoupled from the context of the traditions and communities that nurture such experiences and (2) as a result orphaned and vulnerable either to turning into extravagant forms of isolated beliefs and practices or being subsumed by an exclusivist scientific understanding. The upshot of this is that:

Transpersonal and spiritual knowledge claims are [in danger of only being deemed] valid because they can be replicated and tested through disciplined introspection, and can therefore be intersubjectively verified or falsified.

[Ferrer 2002:42-43]

If such an approach gained ground, it would be a further step in the direction of what has been called "the empiricist colonization of spirituality," something that many of the transpersonal theorists have been unknowingly working towards in spite of their intent and claim to do otherwise (Ferrer 2002:41-70). As Ferrer has persuasively argued, there are other ways to include the spiritual into a transpersonal model of consciousness, and one is to follow the track of the spiritual traditions and cultures themselves that, after all, provide the sources and context for most of the spiritual experiences people have. Instead of the experiential and empirical approaches and their limitations, he advocates for a participatory perspective that is inclusive and pluralistic and is expressed in personal, interrelational, communal, and place-based ways (115ff.)

Here the work that has been undertaken in the broad sub-area of the anthropology of consciousness offers a way of completing the circle of consciousness, from the objective outer to the subjective inner, to the social, to the transpersonal. Edith Turner, in her recently published lecture before the Society for the Anthropology of Consciousness, entitled "Advances in the Study of Spirit Experience: Drawing Together Many Threads," presents a bird's-eye view of the history of the shift in fieldwork practice; it shows an openness to anomalous experiences on the part of a number of anthropologists working in different 
cultures, including one she underwent. In outspoken language and with considerable determination, she lays out the issues, the resistances, and the paradigm shift that some anthropologists experienced when they truly entered into the spiritual worlds of the cultures they were studying. One of her moving examples comes from the late Colin Turnbull. Like Ferrer, he emphasizes the participatory:

To conclude, what is needed for this kind of fieldwork is a technique of participation that demands total involvement of our whole being. Indeed it is perhaps only when we truly and fully participate in this way that we find this essentially subjective approach to be in no way incompatible with the more conventional rational, objective, scientific approach. On the contrary, they complement each other and that complementarity is an absolute requirement if we are to come to any full understanding of the social process. It provides a wealth of data that could never be acquired by any other means.

[Turner 2006:42, citing Turnbull 1990:51]

In the last section of Turner's lecture, entitled "Implications for the Future," she offers the following glance down the road:

There are various trends. Toward spiritualism studies? A serious study of psychics? More on death, consciousness, NDEs, and reincarnation? More dream studies? Multiple personality (seemingly a dead end right now)? The body? Shamanism? Spirits? Theories of ultimate reality-energy, biology? There have been many already. I think we need a big new offensive. The SAC will have to rescue the Society for the Anthropology of Religion from its studies of superficials, to enter the field of religion, and say far more than the SARs seem to have the nerve to say. It is important at last to find out what this psi, this chi, this wakan, this shamanic gift is. We do indeed need to get close to it to know it, and closeness is now of the essence. So far, one of the best ways has been shown to be what we have done, to accumulate full-scale ethnographies on spirituality and psi as those faculties appear in the lives of different people all over the world. When these become commonplace, science itself will be compelled to join up with us and accept the human being as spirit-involved. Once this is achieved, we can together lay out the characteristics of psi, spirits, and their provenance, and become familiar with them.

Perhaps if Turner's agenda is undertaken, we will then begin to know something more profound than we do now about the nature of consciousness and, if so, it will no doubt look rather different from the limited conceptions of it we have seen entertained in the last decades. It will also include other cultures into the larger conversation and find a place for their rich heritage and visionary experience that have for too long been neglected in the arid discussions that have passed for theory and fact. It is high time that our educational institutions considered 
taking on board this more ambitious and more meaningful project in the service of consciousness studies.

\section{CONCLUSION}

To conclude, in our experience and that of the students at Goddard, an inclusive approach to consciousness studies has the potential of addressing more completely the subject of consciousness in all its expressions. This multifaceted approach would have the value of freeing consciousness studies from the hegemony of scientific empiricism, as well as providing a way to steer cautiously through the channel of first-person consciousness and transpersonal experiences. After navigating these areas, a foundation could then be laid for a multidisciplinary and even transdisciplinary perspective where a disciplined pluralism would pervade and all parts of the spectrum of consciousness would be given due consideration, including other perspectives from other cultures and times (Random 1996). It has been part of our experience and privilege at Goddard to be a place where we can pursue some of these perspectives, struggle to include them in our work, and to contribute in our own modest way to the furthering of the incredibly rich experience of "doing consciousness studies."

\section{NOTE}

1. Currently, it has an enrollment of approximately 10 students per semester with the expectation of doubling this in the next few years and has 5 graduates.

\section{REFERENCES}

Cortright, B.

1997 Psychotherapy and Spirit: Theory and Practice in Transpersonal Psychotherapy. Albany: State University of New York Press.

Dalai Lama 2005 The Universe in a Single Atom: The Convergence of Science and Spirituality. New York: Morgan Road Books.

Editor, Journal of Consciousness Studies 1997 The Future of Consciousness Studies (editorial). Journal of Consciousness Studies $4(5-6): 385-388$.

Faw, B.

2006 Are We Studying Consciousness Yet? Toward a Science of Consciousness Conference, April 4-8, 2006, Tucson. Journal of Consciousness Studies, 13(4):81-99

Ferrer, J.

2002 Revisioning Transpersonal Theory: A Participatory Vision of Human Spirituality. Albany: State University of New York Press.

Gadamer, H.G. 1988 Truth and Method. New York: Crossroad. 
Murphy, M., S. Donavan, and E. Taylor 1997 The Physical and Psychological Effects of Meditation, 2nd ed. Sausalito, CA: Institute of the Noetic Sciences.

Random, M., ed. 1996 La Pensée Transdisciplinaire et le Réel. Paris: Editions Devry.

Turnbull, C. 1990 Liminality: A Synthesis of Subjective and Objective Experience. In By Means of Performance, Richard Schechner and Willa Appel, eds. Pp. 50-81. Cambridge: Cambridge University Press.

Turner, E. 2006 Advances in the Study of Spirit Experiences: Drawing Together Many Threads. Anthropology of Consciousness 17(2):33-61.

Varela, F., and J. Shear, eds. 1999 The View from Within: First Person Approaches to the Study of Consciousness. Thorverton, UK: Imprint Academic.

Whitehead, C.

2004 Everything I Believe Might Be a Delusion. Whoa! Tucson 2004: Ten Years On, and Are We Any Nearer to a Science of Consciousness? Journal of Consciousness Studies, 11(12):68-88. 